\title{
LA TERAPIA OCUPACIONAL EN EL ABORDAJE DE LAS ADICCIONES: UNA REVISIÓN ACTUALIZADA
}

\author{
OCCUPATIONAL THERAPY IN ADDRESSING ADDICTIONS: AN UPDATED REVIEW
}

\section{Luzetty Chaves Bazzani'}

\begin{abstract}
Resumen
Con esta revisión se buscó sintetizar los conocimientos actuales sobre el abordaje de las adicciones desde la terapia ocupacional. Se realizó a través de una búsqueda en las bases de datos EMBASE, ISTOR, LILACS y SCIELO, de artículos en el periodo 2003 - 2013 y de publicaciones periódicas internacionales y libros especializados tanto en adicciones como en terapia ocupacional disponibles en línea, con libre acceso en texto completo. Los artículos encontrados hacían referencia a la intervención de terapia ocupacional en adicciones principalmente a drogas, en cuanto al diseño de instrumentos de evaluación y propuestas de intervención. Solamente se identificó en un caso la medición del impacto de los programas. Aunque hay importantes esfuerzos en investigación, resultan insuficientes teniendo en cuenta la magnitud de la problemática y los diversos enfoques desde los cuales es posible abordarla. Se concluye que se requiere estimular la investigación que contribuya al desarrollo conceptual y empírico en el área desde terapia ocupacional.
\end{abstract}

\section{Palabras clave:}

Terapia ocupacional, adicción, abuso de sustancias.

\begin{abstract}
This review seeks to synthesize current knowledge on addressing addictions in occupational therapy. It was conducted through a search of EMBASE, JSTOR, LILACS and SCIELO databases of articles in the period 2003-2013 and international journals and books in both addictions and occupational therapy issues available online, with free and full text. The items found were related to occupational therapy intervention in drug addiction, mainly about the design of assessment tools and intervention proposals. Although significant efforts in researching are evident, those are insufficient given the magnitude of the problem and the various approaches from which is possible address them. Only one case was evident in measuring the impact of occupational therapy programs. It is concluded that research is needed to stimulate the development of conceptual and empirical approach to addiction by occupational therapists.
\end{abstract}

\section{Keywords:}

Occupational therapy, addiction, substance abuse.

1 Terapeuta Ocupacional Universidad Nacional de Colombia. Especialista en Gerencia Social Escuela Superior de Administración Pública ESAP. Bogotá, Colombia.

E-mail: Icbazzani@unal.edu.co Teléfono: 3188780236 


\section{INTRODUCCIÓN}

La problemática de las adicciones, conocidas desde el antiguo Egipto como "enfermedades incurables del alma" (Soto, 2011, p. 21), ha sido uno de los campos en los cuales los terapeutas ocupacionales han tenido tradicionalmente una participación. El Manual Diagnóstico y Estadístico de los Trastornos Mentales DSM-IV-TR (American Psychiatric Association, 2002) refiere que para ser diagnosticada como tal, la adicción a sustancias conlleva un patrón desadaptativo de consumo que genera malestar o deterioro. Tanto la Clasificación Internacional de Enfermedades - CIE-10, como el DSM-IV-TR, establecen una serie de criterios de los cuales señalan que deben darse al menos tres de éstos, durante un periodo de doce meses, para que pueda hablarse de dependencia, como: tolerancia, esfuerzos infructuosos de interrumpir el consumo, síndrome de abstinencia, inversión cada vez mayor de tiempo en actividades relacionadas con la obtención de la sustancia o con la recuperación de sus efectos y persistencia en el uso de la sustancia a pesar de percibir de forma clara sus efectos perjudiciales, entre otros (American Psychiatric Association, 2002; Organización Mundial de la Salud, 2005).

En la actualidad, el término drogodependencia está siendo sustituido por el de adicción, al referirse no solo a los trastornos por dependencia de sustancias químicas, sino también a una diversidad de conductas patológicas "altamente disruptivas para el individuo que las padece que se denominan adicciones comportamentales y que se caracterizan por la necesidad imperiosa de ser repetidas, a pesar del evidente daño psíquico y físico que generan" (Casas, Bruguera, Duro \& Pinet, 2011 , p. 17 - 25). Entre estos otros tipos de adicciones, se reconocen la dependencia a internet, juegos, sexo, compras, videojuegos, trabajo y la dependencia a relaciones afectivas (Pérez \& Martín, 2007; Fuentes, 2008). A pesar de que las nuevas adicciones aún no están clasificadas en el DSM-IV, "presentan la misma sintomatología que las adicciones por sustancias y por tanto, pueden ser tan peligrosas en sus consecuencias como ellas" (Fuentes, 2008, p. 2). Las condiciones de desarrollo de la sociedad postmoderna conllevan al ser humano a una aceptación inconsciente de comportamientos y formas de vida, lo cual obstaculiza la identificación de las adicciones. De hecho, en el marco de las llamadas nuevas adicciones, ciertas conductas pueden pasar inadvertidas, o en otros casos pueden llegar a ser "premiadas" por la sociedad, por ejemplo al tratarse de adicción al trabajo o al sexo. De esta forma se dificulta la percepción que la persona pueda tener acerca del origen de su malestar, y por tanto, puede verse limitado el control que pueda tener hacia sus conductas y sus efectos. Como lo señalan Pérez y Martín:

Al explorar y diagnosticar las nuevas
adicciones hemos podido constatar el
desorden y la confusión en que viven las
personas adictas. Y no se trata de algo
superficial, sino de algo muy profundo y
frustrante que angustia sus vidas, como
si ante tanto dolor y sufrimiento el ser
humano necesitara protegerse como
solución o ruptura y crear una suave
película que, de algún modo, aliviara
sus penas. Y lo que suele suceder es
precisamente lo contrario, que cuanto
más intenta protegerse, más angustia se
produce (Pérez y Martín, 2007, 268).

En este contexto, surge la inquietud acerca de cuáles son los aportes que la terapia ocupacional está brindando en la actualidad con respecto a la problemática de las adicciones, que sin duda ocasiona importantes alteraciones en las actividades de la vida diaria, los roles, hábitos, y en general, en el desempeño ocupacional de las personas. Por lo tanto, se pretende con esta revisión, identificar y sintetizar los conocimientos actuales sobre el abordaje de las adicciones desde la terapia ocupacional, con el fin de facilitar la actualización de los profesionales en este campo y finalmente, identificar nuevas oportunidades de investigación.

\section{MÉTODO}

Se realizó la revisión en las bases de datos EMBASE, JSTOR, LILACS y SCIELO, con la búsqueda de artículos originales y de revisión que se encontraban en el periodo de 2003 a 2013, y con combinaciones de los descriptores: terapia ocupacional, adicción y abuso de sustancias. Adicionalmente se realizó la búsqueda en publicaciones periódicas internacionales y libros especializados tanto en adicciones como en terapia ocupacional disponibles en línea y con libre acceso. Se delimitó a los idiomas español, inglés y portugués y se consideró que los artículos obtenidos tuvieran en su título y en su resumen la información pertinente del tema, excluyendo artículos no disponibles en texto completo. 


\section{Resultados}

A partir de la revisión realizada se encontraron 96 artículos, de los cuales solo 22 hacían referencia a la intervención de terapia ocupacional en adicciones. De estos 22 productos se encontró que provenían principalmente de España y Chile (12 y 5 artículos respectivamente). En cuanto a los autores, 16 de los documentos fueron realizados por terapeutas ocupacionales; los 6 restantes, de otras disciplinas, relacionaban experiencias o presentaban enfoques en los cuales se consideraba a la terapia ocupacional como parte del proceso. Con excepción de 3 artículos que hacían referencia a la dependencia del alcohol, los documentos identificados se centraban en la dependencia a las drogas, y en ningún caso a adicciones no químicas o comportamentales. Los estudios liderados por profesionales de terapia ocupacional correspondían principalmente a investigaciones cualitativas, especialmente a nivel de diseño de instrumentos de evaluación y propuestas de intervención. En la búsqueda realizada, solamente se evidenció en un caso la medición del impacto de los programas de rehabilitación que incluyen a la terapia ocupacional en la población con adicciones.

\section{Rol del terapeuta ocupacional en adicciones}

El terapeuta ocupacional, como profesional que interviene en la rehabilitación de adicciones, tiene unas funciones definidas de acuerdo a los desarrollos conceptuales de la profesión. Sin embargo, en muchos contextos estas funciones no son muy conocidas, y el impacto de la intervención de dicho profesional no ha sido evidenciado. Esto lo refiere Rojo, terapeuta ocupacional española, quien afirma que el rol asignado a los terapeutas ocupacionales ha sido en general secundario y orientado a mantener ocupadas a las personas con adicciones. La autora señala que la responsabilidad de esta situación es en parte, de los propios terapeutas por la dificultad que han tenido para transmitir su marco de trabajo, su rol y sus espacios de intervención: "se diría que no hemos sido capaces de transmitir la diferencia entre «mantener ocupado» y «trabajar científicamente la ocupación humana»" (Rojo, 2008, p. 88-97). En relación a esta situación, Díaz y Palucci (2010) realizaron en un estudio referente al papel de los diferentes profesionales que laboran en centros de atención a drogas en Bogotá, encontrando que en el caso de los terapeutas ocupacionales, para la muestra seleccionada corres- pondiente a cinco profesionales, no se evidenciaron funciones como la de inserción social que se enuncia en su perfil profesional.

En cuanto a las acciones desempeñadas, Riveros realizó en Chile una investigación en la cual se utilizó el instrumento Entrevista Histórica de Funcionamiento Ocupacional (OPHI-II), orientado a construir un perfil ocupacional del consumidor de drogas. Se encontró que "existe cierto grado de disfunción ocupacional en las tres escalas que conforman el instrumento (identidad ocupacional, competencia ocupacional y ambientes de comportamiento ocupacional), lo que a su vez es posible de asociar con el consumo de drogas" (Riveros, 2003, p. 48-58). Se concluye que es fundamental conocer, evaluar e intervenir el desempeño ocupacional "en etapas tempranas, como parte de un programa de prevención que pretenda ser exitoso en evitar el inicio del consumo de drogas".

Crouch (2007). ofrece una descripción del impacto que tienen las adicciones en el desempeño ocupacional de las personas, teniendo en cuenta las actividades de trabajo, de la vida diaria, ocio y tiempo libre, y el impacto en roles, hábitos y rutinas. Respecto al trabajo, la autora señala que el efecto de la dependencia a las sustancias sobre el desempeño laboral de la persona implica importantes costos para la familia, el empleador y la sociedad al asociarse con el ausentismo, el presentismo, los accidentes, la disminución de la productividad, entre otros. Respecto a las actividades de la vida diaria, señala que puede haber disminución o pérdida de la habilidad de la persona para realizar sus actividades de autocuidado y cuidado del ambiente. En el caso de las mujeres, "sus casas son a menudo impecables, y las necesidades físicas de la familia se encuentran bien atendidas, sin embargo, las necesidades emocionales de la familia son atendidas de una manera deficiente" (Crouch, 2007, p. 186). En lo referente a las actividades de ocio y tiempo libre, Crouch plantea que comúnmente las personas dependientes de sustancias no tienen hobbies o pasatiempos, más que el mismo uso de la sustancia. Señala que la mayoría de éstos se asocian con el uso del alcohol y las drogas, por lo cual, un objetivo fundamental en la intervención sería orientar hacia un uso significativo del tiempo libre.

En relación al impacto sobre los roles, hábitos y rutinas, Crouch afirma que los desórdenes por el uso de sustancias conllevan a una pérdida del balance entre el trabajo, el descanso y la diversión. "La persona no emplea sus capacidades al máximo, presentándose un desbalance y fallas para desarrollar o mantener el 
funcionamiento normal en la mayoría de ocupaciones. Esto lleva a aislamiento social, menos tiempo en el trabajo y con la familia, y ruptura en los sistemas de apoyo y las relaciones" (Crouch, 2007, p. 187).

\section{Evaluación de terapia ocupacional}

En cuanto a la evaluación de terapia ocupacional en adicciones, se identificaron propuestas provenientes de Sudáfrica, España y Chile. En Sudáfrica se plantean lineamientos de evaluación para el tratamiento de las adicciones, que constan de: entrevistas, observaciones clínicas, Evaluación de Estrés (Crouch 2003), Hospital Anxiety and Depression Scale, Inventario de Depresión de Beck y la Medición Canadiense del Desempeño Ocupacional (Crouch 2007).

Rojo (2008), plantea desde el Modelo de la Ocupación Humana, una propuesta de evaluación que incluye:

1. Entrevista Histórica del Desempeño Ocupacional OPHI-II. (Kielhofner, 1998).

2. Cuestionario Ocupacional (OQ): Autorregistro de las actividades que se desempeñan a lo largo de una semana.

3. Listado de roles (Oakley, Kielhofner, 1986): Mide roles desempeñados en el pasado, en la actualidad y lo que le gustaría desempeñar en el futuro.

4. Listado de intereses (Kielhofner, Neville, 1983): Consiste en un inventario en el que se solicita al entrevistado que gradúe su interés en cada una de las actividades en el pasado, si participa actualmente en esa actividad y su deseo de realizarla en el futuro.

5. Cuestionario Volicional (VQ) (Chern, Kielhofner, 1996): Aporta información sobre motivación e impacto del medio ambiente sobre la volición.

6. Evaluación de habilidades motoras y de procesamiento: (Assessment of Motor and Process Skills, AMPS; Fisher, 2003). Es un sistema de evaluación protocolizado que tiene como objetivo valorar la calidad del desempeño en actividades de la vida diaria.

La misma autora (Rojo, 2011) propuso el Autoinforme de Desempeño Ocupacional ADO, un cuestionario adaptado del Occupational Self Assessment -OSA-de Kielhofner (2009), y validado específicamente para población con adicción a drogas, que permite la autoevaluación del funcionamiento ocupacional y la influencia del ambiente en el desarrollo de las actividades de la vida diaria de las personas que realizan un tratamiento para abandonar sus hábitos adictivos.

Por otra parte, en Latinoamérica, el Programa de integración socio-ocupacional para personas drogodependientes del Consejo Nacional para el Control de Estupefacientes (2004) en Chile, propone pautas de evaluación, modificadas del Modelo de Ocupación Humana. En otro estudio realizado en este mismo país (Farías, Guerra, Cifuentes, Rozas \& Riveros, 2010) se exploraron los componentes ocupacionales que los terapeutas evalúan durante el proceso de tratamiento del consumo de drogas. Los resultados señalaron que la mayoría de los profesionales evalúan los componentes de rutina/hábitos, roles, volición, tiempo libre e historia laboral en la etapa de ingreso a Centros de Tratamiento y Rehabilitación. También se identificó una "carencia de modelos y evaluaciones adecuadas y específicas desde la Terapia Ocupacional en el tema de estudio y carencia de homogeneidad en el lenguaje, instrumentos de evaluación y los modelos utilizados entre terapeutas ocupacionales" (p. $45-56$ ).

\section{Tratamiento de terapia ocupacional en adicciones}

En el Modelo Canadiense del Desempeño Ocupacional, basado en una perspectiva centrada en el cliente, "la mayoría de definiciones se relacionan con una fuerza de vida motivadora" (Simó \& Urbanowsky, 2006). Esto hace referencia a la necesidad de buscar la motivación desde la perspectiva de la persona, en cuanto a identificar aquello que le da sentido a su vida y desde lo cual la persona puede encontrar fuerzas para luchar. El autor ejemplifica este supuesto, a partir de una experiencia en una cárcel, con mujeres que presentaban adicción, cuyo proyecto de intervención "se basó en el rol de madres, ya que sus hijos eran su principal fuerza de vida y motivación, para enfrentar el encarcelamiento y la adicción" (Simó \& Urbanowsky, 2006). Cáceres y Mesías (2011) refieren que en el Instituto de Adicciones de Madrid, la terapia ocupacional tiene como uno de sus objetivos "la motivación y contención, utilizando la actividad como una herramienta terapéutica para la vinculación al tratamiento de forma que facilite el descubrimiento de nuevos intereses 0 reencuentro con aquellos perdidos" (p. 226 - 34).

Por otra parte, según Punanova y Petrova (2009) el objetivo principal de la intervención de terapia ocupacional es aprender a volver a la vida normal. El profe- 
sional puede intervenir en los ambientes terapéuticos basados en el concepto de los programas de doce pasos, como lo señalan las autoras, en una experiencia de tratamiento de personas dependientes de drogas y alcohol en Rusia en las Ilamadas "sociedades terapéuticas". Las autoras citan a Wallen (1987), para describir los programas de doce pasos, señalando que en un primer momento, las personas admiten que tienen una enfermedad, que desean recuperarse, posteriormente aceptan la ayuda del equipo, y analizan su propio comportamiento, incluyendo todos los fallos y daños infligidos a otras personas y finalmente, realizan autocontrol y buscan modificar su comportamiento. Este programa se lleva a cabo en un ambiente artificial, que para facilitar la abstención de drogas o alcohol, debe estar libre de factores desencadenantes. Luego de completar el programa de tratamiento, la autoras señalan que alrededor del $8 \%$ al $10 \%$ de los pacientes regresan para un tratamiento de prevención de recaídas, y el 15\% tienen recaídas periódicas pero con largos períodos de sobriedad y una mejor calidad de vida (Punanova \& Petrova, 2009). El desempeño del terapeuta ocupacional dentro de las comunidades terapéuticas, es también referido en España por Llorente, Mezo y Barrutia (2011).

En Brasil, Antoniassi, Leal y Tedesco afirman que entre los puntos clave en la intervención con drogodependientes, se destacan las conductas de riesgo y "las pérdidas o daños que el uso de las sustancias pueden generar en las diferentes esferas de sus vidas ocupacionales" (2008, p. 221- 8). Las autoras señalan además, que las experiencias de terapia ocupacional no están siendo reportadas en la literatura. Finalmente, en lo relacionado con la rehabilitación de personas dependientes del alcohol, la intervención de terapia ocupacional es reportada por Kiritzé, Huas, Rosenzweig, Comte, Paille \& Lehert (2006) en Francia, por Kopelman, Thomson, Guerrini, \& Marshall (2009) en el Reino Unido y por Santi (2001) en España, en programas con características similares a los de rehabilitación de la dependencia a drogas.

\section{Neurociencias, terapia ocupacional y trastornos adictivos}

En los últimos años, se ha profundizado el abordaje de las adicciones desde el punto de vista de las neurociencias, con investigaciones acerca de los mecanismos neurobiológicos que intervienen en el inicio, mantenimiento y recaídas de las adicciones, resaltándose la labor del terapeuta ocupacional en este campo (Pedrero, Ruiz,
Verdejo, Llanero \& Ambrosio, 2011). El aporte de esta profesión se ha evidenciado en propuestas como la intervención en la rehabilitación de la disfunción ejecutiva en adictos a sustancias por Rojo, Pedrero, Ruiz, Llanero, Olivar \& Puerta (2009) y en la evaluación del desempeño ocupacional "basada en la función, centrada en las actividades de la vida diaria desde los aspectos globales del comportamiento hasta los sustratos neurológicos y neuropsicológicos que lo sustentan" (Rojo, Sánchez, Gómez \& Martínez, 2011; Rojo, Iraurgi \& Sánchez, 2011). Los autores plantean un protocolo de evaluación orientado al diseño de un tratamiento basado en el Modelo de Rehabilitación Cognitiva Funcional de Lee, Powel y Esdaile (2001). Se propone además, un protocolo para la evaluación neuropsicológica de las adicciones (Ruiz, Pedrero, Rojo, Llanero \& Puerta, 2011), que retoma test neuropsicológicos, escalas de sintomatología cotidiana y pruebas de desempeño ocupacional. Para la detección de alteraciones en las funciones neurocognitivas, se validó en España la evaluación cognitiva de Montreal (MOCA) en personas adictas (Rojo, Pedrero, Ruiz, Llanero \& Puerta, 2013).

\section{Abordaje hacia la inclusión sociolaboral}

Desde otro enfoque, con el Programa de integración socio-ocupacional para personas drogodependientes, el Consejo Nacional para el Control de Estupefacientes (CONACE) de Chile (2004), define pautas de evaluación a partir del Modelo de Ocupación Humana. Se tiene en cuenta además el Modelo Biopsicosocial y el Modelo de Integración Socio-ocupacional desde el cual adaptan herramientas de evaluación para población farmacodependiente. Los instrumentos que se proponen desde esta perspectiva son la Ficha autoaplicada socioocupacional del Programa Alcohol y drogas y la Entrevista Histórica del Desempeño Ocupacional adaptada para su aplicación en adicciones, la cual corresponde a una entrevista semiestructurada modificada de la Entrevista para la Integración Socio-ocupacional, que es a su vez una modificación para su uso en adicciones a partir del OPHI - II Entrevista Histórica del Desempeño Ocupacional. A partir de estos desarrollos en Chile, Schliebener (2012) presentó una propuesta de intervención en el programa alcohol y drogas. Salgado (2012) por su parte, propuso en el mismo año el Programa de intervención para el Apresto Laboral de Personas con Consumo Problemático de drogas, el cual ofrece herramientas de intervención específicas a nivel de preparación laboral. 


\section{Abordaje de la familia}

Las interacciones entre la persona que presenta una adicción y quienes viven con ella, por lo general produce tensiones y emociones negativas. En muchos casos se desarrolla una relación patológica de codependencia, en la cual "la actitud fundamental consiste en un intento de ayuda al adicto, aun a costa del propio sacrificio. Esto hace que el codependiente - coadicto se convierta en un cómplice de la enfermedad y contribuya a perpetuarla" (Fuertes \& Maya, 2005, p. 89). En este sentido, Santi (2006) señala que el terapeuta ocupacional puede aportar en la identificación de estas situaciones y participar en la elaboración de un plan de trabajo, con el fin de orientar a la familia. La autora señala que esto es fundamental ya que en ocasiones la familia se convierte en una barrera para el tratamiento, más que un facilitador, al intentar acelerar el proceso de reinserción de la persona adicta a su medio familiar - social cotidiano o desarrollar una variedad de conductas codependientes que dificultarán su recuperación. Según la propuesta de Santi, el plan de trabajo de terapia ocupacional con la familia de personas con alcoholismo, puede incluir los objetivos como: promover una rutina sana y equilibrada que permita a la familia fortalecerse para la futura reinserción de la persona en su núcleo, trabajar para establecer objetivos realistas, orientar a la familia para que reciba apoyo de grupos para-familiares de personas con adicciones (Al Anón, Codependientes anónimos CODA) y apoyar para que cada miembro de la familia retome sus roles y se compartan responsabilidades.

\section{DISCUSIÓN}

A partir de las dinámicas sociales en la actualidad es posible evidenciar una creciente presencia de insatisfacción, ansiedad, falta de autoestima tanto a nivel individual como colectiva, y es necesario reconocer que todo esto, en su conjunto, puede facilitar el desarrollo de las adicciones. La participación de los terapeutas ocupacionales en este ámbito se ha dado desde hace ya varias décadas y se han evidenciado importantes avances. Sin embargo, es necesario profundizar en la construcción conceptual con el fin de que los profesionales logren proyectar la relación entre los conceptos y filosofía propia de la terapia ocupacional y la práctica profesional en el campo de las adicciones.

Los fundamentos filosóficos de la terapia ocupacional, frente a la profunda desestructuración de las acti- vidades cotidianas, y la alteración de los roles, hábitos y rutinas que implican la presencia de una adicción, constituyen una oportunidad para encontrar el sentido de la vida en el desempeño de las ocupaciones. Retomar el sentido personal y social que tiene la ocupación con propósito, recordar que la ocupación desde un sentido espiritual da una identidad y conlleva a la realización humana y valorar la importancia subjetiva que tienen determinadas ocupaciones para cada persona, son las tareas que el terapeuta ocupacional debe llevar a cabo en su práctica cotidiana. Como lo afirman Trujillo, Sanabria, Carrizosa y Parra, la ocupación es vista como un "factor a través del cual el ser humano puede desarrollar sus potenciales para hacerse sujeto, gestor y consultor de su propia historia" y enfatizan en su potencial "para el desarrollo, la autorreflexión y la trascendencia del ser" (2011). La ocupación tiene el potencial de permitir la interacción del ser humano con su entorno y con su propia esencia espiritual, y de esta forma, promover el logro de profundas transformaciones en su existencia. Esto también es reafirmado por Moruno y Romero (2004):

\section{El terapeuta ocupacional utiliza o aplica en su práctica clínica, diversas actividades que constituyen una unidad cultural y personalmente significativa; aquellas que comprometen al individuo, en las que se implica y se envuelve, aquellas a través de las que se distingue y expresa, aquellas que además de dar respuesta a las necesidades vitales y requerimientos sociales se constituyen en una forma de dar sentido a la existencia, aquellas que contribuyen a construir la identidad personal, social y cultural.}

A nivel del desarrollo investigativo de la terapia ocupacional en el campo de las adicciones, se evidencian importantes esfuerzos que, sin embargo, resultan insuficientes teniendo en cuenta la magnitud de la problemática y los diversos enfoques desde los cuales es posible abordarla. Las investigaciones realizadas se han centrado en aspectos psicobiológicos, y se han dado avances importantes a nivel de inclusión sociolaboral y en la caracterización del desempeño ocupacional. Desde el área laboral, el terapeuta ocupacional actúa como facilitador de los procesos de prevención, rehabilitación e inclusión en diversas problemáticas sociales. En este contexto, la preparación laboral de personas 
dependientes es un importante campo para ahondar en la investigación.

Teniendo en cuenta los fundamentos filosóficos y conceptuales de la profesión, es necesario profundizar en la estructuración teórica del abordaje de las adicciones y desarrollar propuestas de intervención que permitan evidenciarlo en la práctica. El terapeuta ocupacional debe tener especial precaución en su quehacer, evitando proyectar roles como el de instructor de manualidades o promotor de actividades para ocupar el tiempo y evidenciar la orientación de su intervención hacia el autodescubrimiento y el desempeño de ocupaciones verdaderamente transformadoras para cada sujeto. Por otra parte, es necesario explorar las posibilidades de encuentro entre las diferentes propuestas y avances en el manejo de la dependencia a las drogas, con respecto a la generalidad de las adicciones, como la dependencia al alcohol y las adicciones comportamentales. La indagación del potencial de la ocupación para la prevención de los trastornos adictivos es otro campo de gran relevancia, en especial para el trabajo con la infancia y la adolescencia. En todos los casos, es fundamental promover la documentación de las experiencias y la medición del impacto de los programas de terapia ocupacional en adicciones.

\section{Conclusiones}

A partir de la revisión realizada es posible identificar la necesidad de que la academia estimule la investigación que contribuya al desarrollo conceptual y empírico del abordaje de las adicciones por parte de los terapeutas ocupacionales. Aunque se evidencian importantes esfuerzos, resultan insuficientes teniendo en cuenta la magnitud de la problemática y los diversos enfoques desde los cuales es posible abordarla. En la medida en que se promueva la investigación y su difusión en la comunidad profesional, será posible que los terapeutas ocupacionales logren proyectar de una manera más contundente la relación entre los conceptos y filosofía propia de la profesión y la práctica cotidiana en la problemática de las adicciones. Así mismo, se requiere propender por desarrollar la cultura de la documentación de las experiencias y la evaluación de programas, que permitan continuar el desarrollo de herramientas propias de terapia ocupacional y promocionar el empleo y validación de las ya existentes. Establecer redes entre grupos de investigación interesados en la temática de las adicciones facilitará el intercambio de experiencias y la generación de conocimientos. La sociedad, en las condiciones actuales, inevitablemente seguirá facilitando el desarrollo de adicciones. La ocupación con propósito da una identidad y conlleva a la realización humana. Son dos afirmaciones que demandan la acción oportuna y responsable del terapeuta ocupacional. Pero más que eso, demandan la búsqueda espiritual del profesional frente al sentido personal y social de su propia ocupación.

\section{REFERENCIAS BIBLIOGRÁFICAS}

American Psychiatric Association (2002). DSM-IV-TR Manual diagnóstico y estadístico de los trastornos mentales. $4^{\mathrm{a}} \mathrm{ed}$. Washington.

Antoniassi D.; Leal J. \& Tedesco S. (2008). Terapia ocupacional e farmacodependência: categorização e atualização das publicações nacionais. Revista O Mundo da Saúde, 32(2), 221- 8.

Cáceres A. y Mesias B.( 2011). Atención a Población sin Hogar: Experiencia del Instituto de Adicciones de la Ciudad de Madrid. En: J. Bobes, M. Casas y M. Gutiérrez. Manual de Trastornos Adictivos (pp. 226 - 34). Madrid, España: Enfoque Editorial S.C.

Casas M.; Bruguera E.; Duro P. y Pinet C. (2011). Conceptos básicos en trastornos adictivos. En: J. Bobes, M. Casas \& M. Gutiérrez. Manual de Trastornos Adictivos. (pp. 17 - 25). Madrid, España: Enfoque Editorial S.C.

CONACE - Consejo Nacional para el Control de Estupefacientes. (2004). Abriendo Puertas. Programa de integración socio-ocupacional para personas drogodependientes. Gobierno de Chile.

Crouch R. (2007). Substance Use Disorders. Occupational Therapy Prescribed Minimum Benefits. Occupational Therapy Guidelines Mental Health. South African Society of Psychiatrists. SASOP. (p. 187). Accesado el 5 de marzo de 2013. Disponible en: http://www.sasop.co.za/images/OccupationaltherapyGuidelines_MentalHealthPMBs2008.pdf

Díaz L. y Palucci M. (2010). El papel de los profesionales en centros de atención ambulatoria en drogas en la ciudad de Bogotá, Colombia. Revista Latino-Americana de Enfermería, 18, 573 -81.

Farías L.; Guerra V.; Cifuentes T.; Rozas S. y Riveros M. (2010). Consumo problemático de drogas y Terapia Ocupacional: Componentes ocupacionales evaluados durante el proceso de tratamiento y rehabilitación. Revista Chilena de Terapia Ocupacional, 10, $45-56$.

Fuentes M. (2008). Nuevas Adicciones. Ponencia. Congreso Sociedad de Psicoterapia y Psicoanálisis del Centro. (p. 2): A.C. México. 
Fuertes M. y Maya M. (2005). Atención a la familia: la atención familiar en situaciones concretas. Abordaje familiar en consumidores abusivos de alcohol. Anales del Sistema Sanitario de Navarra, 24 (2), 89.

Kiritzé P., Huas D., Rosenzweig C., Comte S., Paille F. \& Lehert P. (2006). A Pragmatic Trial Of Acamprosate In The Treatment Of Alcohol Dependence In Primary Care. Alcohol and Alcoholism, 39 (6), $520-7$.

Kopelman M., Thompson A., Guerrini I. \& Marchall E. (2009). The Korsakoff Syndrome: Clinical Aspects, Psychology and Treatment.: Alcohol y Alcoholism. 44(2), 148-54.

Llorente J., Mezo M. y Barrutia I. (2011). Comunidades Terapéuticas. En: J. Bobes, M. Casas y M. Gutiérrez. Manual de Trastornos Adictivos (pp. 293 - 300). Madrid, España: Enfoque Editorial S.C.

Moruno P. y Romero D. (2004). Terapia ocupacional en salud mental: la ocupación como entidad, agente y medio de tratamiento. Revista Gallega de Terapia Ocupacional, 1.

Organización Mundial de la Salud (2005). Clasificación Internacional de Enfermedades. Décima Versión, CIE - 10.

Pedrero E., Ruiz J., Verdejo A., Llanero M. y Ambrosio E. (2011). El enfoque neurocientífico de las adicciones: Estado actual de conocimientos y perspectivas de futuro. En: E. Pedrero, J. Sánchez, A. Verdejo, M. Llanero y M. Ambrosio (Eds.) Neurociencia y Adicción (pp. 417- 24). Madrid, España: Sociedad Española de Toxicomanías.

Pérez F. y Martín I. (2007). Nuevas Adicciones: ¿Adicciones Nuevas? Alcalá de Henares, (p. 268). España: Intermedio Editores.

Punanova N. \& Petrova T. (2009). Behavioral Approach to Rehabilitation of Patients with Substance-Use Disorders. En: I. Söderback, (Ed.). International Handbook of Occupational Therapy Interventions. (p. 277). Estocolmo, Suecia: Springer.

Riveros M. (2003). Perfil Ocupacional del Consumidor de Drogas. Revista Chilena de Terapia Ocupacional, 3, 48-58. Accesado el 5 de marzo de 2013. Disponible en: http://www.revistaterapiaocupacional.uchile.cl/index.php/RTO/article/viewFile/151/132

Rojo G. (2008). Terapia Ocupacional en el Tratamiento de las adicciones. Revista Trastornos adictivos, 10 (2), 88- 97.

Rojo G., Iraurgi I. y Sánchez A. (2011). Rehabilitación cognitiva en el Tratamiento de las Adicciones. En: E. Pedrero, J. Sánchez, A. Verdejo, M. Llanero y M. Ambrosio (Eds.). Neurociencia y Adicción. (pp. 277-97). Madrid, España: Sociedad Española de Toxicomanías.

Rojo G., Pedrero E., Ruiz J., Llanero M., Olivar A. y Puerta C. (2009). Terapia Ocupacional en la rehabilitación de la disfunción ejecutiva en adictos a sustancias. Revista Trastornos Adictivos, 11 (2), 96 -105.

Rojo G., Pedrero E., Ruiz J., Llanero M. y Puerta C. (2011). Evaluación del Desempeño Ocupacional en la vida cotidia- na en adictos. Creación de un instrumento de medida: el ADO. Revista Adicciones, 23 (1), 27- 35. Accesado el 4 de marzo de 2013. Disponible en: http://www.redalyc.org/ pdf/891/289122829004.pdf

Rojo G., Pedrero E., Ruiz J., Llanero M. y Puerta C. (2013). Cribado neurocognitivo en adictos a sustancias: la evaluación cognitiva de Montreal. Revista de Neurología, 56(3), 129 - 36.

Rojo G., Sánchez A., Gómez C. y Martínez R. (2011). Evaluación del Desempeño Ocupacional en las Adicciones (pp. 215 - 32). En: Neurociencia y Adicción. E. Pedrero, J. Sánchez, A. Verdejo, M. Llanero y M. Ambrosio (Eds.) Madrid, España: Sociedad Española de Toxicomanías.

Ruiz J., Pedrero E., Rojo G., Llanero M. y Puerta C. (2011). Propuesta de un protocolo para la evaluación neuropsicológica de las adicciones Revista Neurología. 53, 483 - 93.

Salgado L. (2012). Programa de intervención para el Apresto Laboral de Personas con Consumo Problemático de drogas. Aproximándose al rol de trabajador. ContexTO. Propuestas para la acción en Terapia Ocupacional, 1(1), 23 - 77. Accesado el 4 de marzo de 2013. Disponible en: http://www.ucentral.cl/prontus_ucentral/site/artic/ 20120801/asocfile/20120801123807/ contexto_n_1.pdf

Santi A. (2006). Alcoholismo: Integración familia - paciente desde la terapia ocupacional. Revista Gallega de Terapia Ocupacional, 4, 23.

Schliebener M. (2012). COSAM Quinta Normal: Propuesta de intervención de T.O. en Programa Alcohol y Drogas Modalidad ambulatorio intensivo a partir de una experiencia práctica. ContexTO. Propuestas para la acción en Terapia Ocupacional, 1(1), 78 - 131. Accesado el 4 de marzo de 2013. Disponible en: http://www.ucentral.cl/prontus_ ucentral/site/ artic/20120801/asocfile/20120801123807/contexto_n_1.pdf

Simó S. (2006). Urbanowsky R. El Modelo Canadiense del Desempeño Ocupacional. Revista Terapia Ocupacional Galicia (3).

Soto C. (2011). Psicoanálisis aplicado al tratamiento de adicciones en comunidades terapéuticas. ¿Posible o imposible? Tesis para optar al título de Magíster en Psicología clínica. Universidad de Chile.

Trujillo A.; Sanabria L., Carrizosa L. y Parra E. (2011). Ocupación: Sentido, realización y libertad. Diálogos ocupacionales en torno al sujeto, la sociedad y el medio ambiente. Rojas (Ed.). Bogotá: Universidad Nacional de Colombia. 